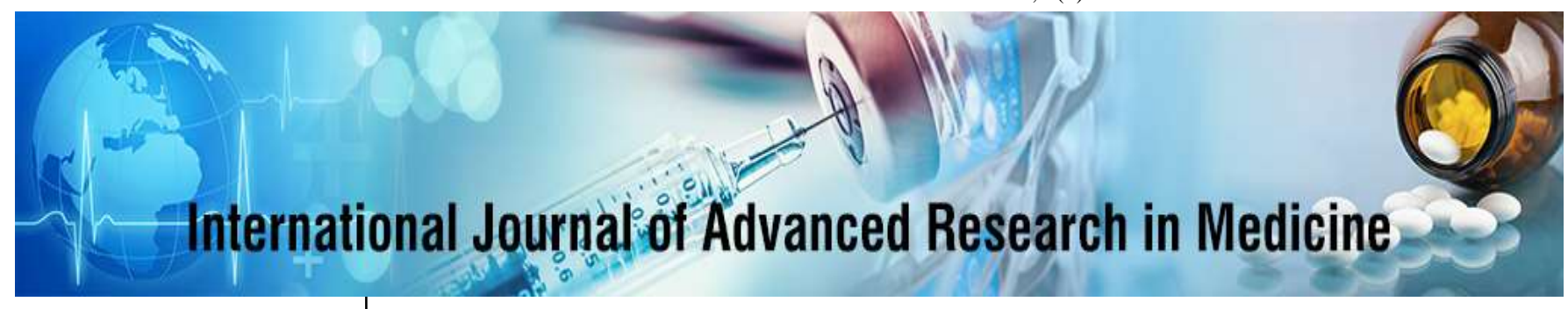

E-ISSN: 2706-9575

P-ISSN: 2706-9567

IJARM 2019; 1(2): 38-40

Received: 15-05-2019

Accepted: 18-06-2019

\section{Rakhi Ludam}

Associate Professor, Department of Pulmonary Medicine, IMS and SUM hospital, Siksha " $O$ "

Anusandhan University, K8, Kalinganagar, Bhubaneswar, Odisha, India

\section{Rohit Mohavarkar}

Department of Pulmonary

Medicine, IMS and SUM

hospital, Siksha "O"

Anusandhan University, K8,

Kalinganagar, Bhubaneswar,

Odisha, India

\section{Banani Jena}

Department of Pulmonary

Medicine, IMS and SUM

hospital, Siksha "O"

Anusandhan University, K8,

Kalinganagar, Bhubaneswar,

Odisha, India

\section{Sangita Jena}

Department of Pulmonary

Medicine, IMS and SUM

hospital, Siksha "O"

Anusandhan University, K8, Kalinganagar, Bhubaneswar, Odisha, India

\title{
Role of BAL fluid CBNAAT for diagnosis of tuberculosis: Our experience at a tertiary care Hospital
}

\author{
Rakhi Ludam, Rohit Mohavarkar, Banani Jena and Sangita Jena
}

DOI: https://doi.org/10.22271/27069567.2019.v1.i2a.92

\begin{abstract}
Background: Sputum smear negative tuberculosis can be a diagnostic challenge and there can be a dilemma in initiation of treatment, CBNAAT is one important test which gives quick results within hours plus an additional information about resistance with rifampicin, in sputum negative or nonexpectorating patients brochoalveoloarlavagae can be useful, if tested for CBNAAT made available via fiberoptic bronchoscopy.

Materials and Methods: Clinico-radiological suspects of pulmonary tuberculosis who showed clinical symptoms and radiological opacities consistent with tuberculosis who were sputum negative or not expectorating sputum were included in this study with exclusion patients in whom bronchoscopy was contraindicated, smear and CBNAAT examination was done of the obtained sample. The data was then analyzed.

Results: in our study of 100 patients 57 were males and 43 were females, the most common lesion detected in imaging was consolidation $44 \%$ of patients followed by cavitary lesions at $27 \%$. Out of 100 patients who were clinicoradiologically suspected for PTB who underwent bronchoscopy 45 were diagnosed PTB out of which 32 tested positive for CBNAAT, no rifampicin resistance was detected in those cases.

Conclusion: CBNAAT obtained from broncho-alveolar samples can be of great value especially when there is a physicians dilemma to start antitubercular treatment, as there are also other conditions mimicking tuberculosis both radiologically and clinically. Hence, BAL results with CBNAAT can help to arrive at a conclusion.
\end{abstract}

Keywords: Tuberculosis, CBNAAT, ZN stain, BAL fluid, Rifampicin

\section{Introduction}

Tuberculosis (TB), one of significant infectious disease which can be avoided and treated, yet causing death, and is a critical health problem around the world. Around the globe, approximately count of "10 million" (ranging "9.0 to 11.1 million") people contracted "Tuberculosis" in 2018, this count has been more or less same in recent years. "Direct sputum smear microscopy" continues to be crucial tool of diagnosis, but might turn out negative up to $50 \%$ case of active pulmonary TB. In patients who are strong suspects of PTB and yet are sputum smear negative, who are a diagnostic challenge fiberoptic bronchoscopy is considered a good option, thought sensitivity of BAL fluid for AFB is low. Though mycobacterial culture is considered gold standard, time taken for results is 2-6 weeks which leads to delated inititation of treatment CBNAAT, a programmed instrument that has certain fundamental properties i.e., "sample processing", "nucleic acid amplification", and "detection of the target sequences in simple or complex samples using real-time PCR and reverse transcriptase PCR".

Results are accessible in below two hours. The accelerated finding of "Mycobacterium tuberculosis" and "resistance" to $1^{\text {st }}$ line drug "Rifampicin" (RIF's) facilitates the treating doctor to take important decisions in the treatment of patient in regards to treatment in the same visit.

Corresponding Author: Rakhi Ludam Associate Professor, Department of Pulmonary Medicine, IMS and SUM hospital, Siksha "O" Anusandhan University, K8, Kalinganagar, Bhubaneswar, Odisha, India

\section{Materials and methods}

This study was done to evaluate diagnostic value of CBNAAT of BAL fluid samples obtained through fiber optic bronchsocopy for an early diagnosis of pulmonary tuberculosis in patients whose sputum samples are negative for AFB and nonexpectorating patients Patients who are suspected cases of PTB clinically (hemoptysis, fever, weightloss, loss of 
apetite) Patients who are suspected cases of PTB radiologically (cavitating lesions, consolidation, infiltrating lesions, military shadows) Age 15-90 years both male and females. Patients who are children age $<15$ years or $>90$ years, sputum smear positive cases of PTB, patients unfit for bronchoscopy.

Results: Out of 100 patients who were cases of preseumptive TB undervent bronchoscopy and BAL fluid was obtained. 32 patients tested positive for CBNAAT and all were rifampicin sensitive, treatment for was started them. 51 patients were confirmed to be diagnosed as non TB on basis of culture or ther tissue diagnosis, 13 patients showed good response to ATT both clinically and radiologically who were not positive for CBNAAT, and 3 patients lost to follow up and 1 patient died during course of disease. All the symptoms of the patients are documented (Fig 1), The types of the TB patterns also noted (Fig 2)

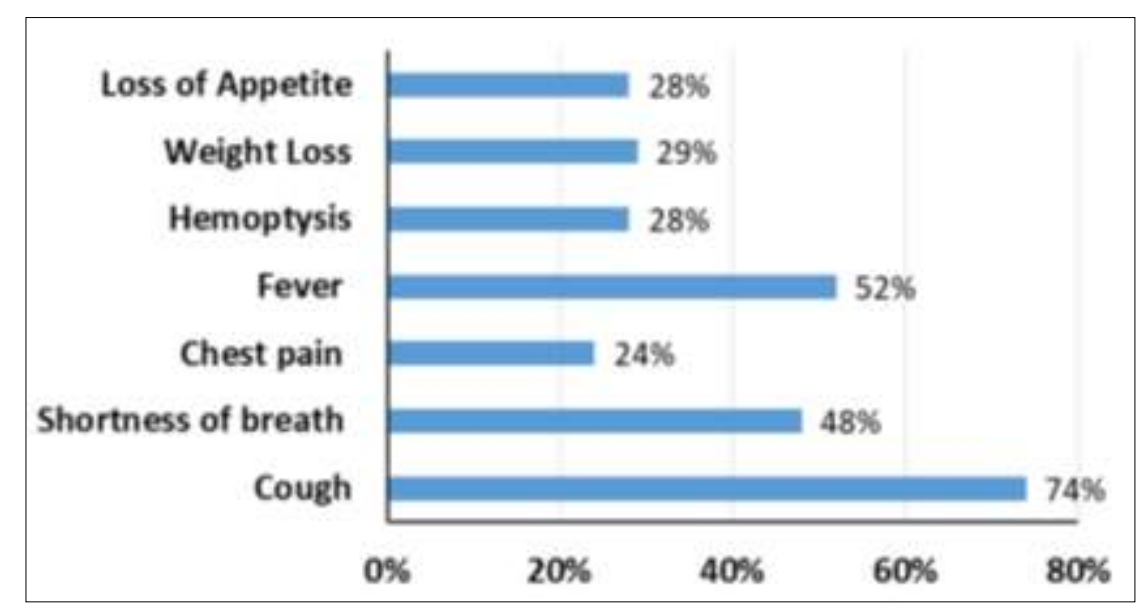

Fig 1: Shows symptoms

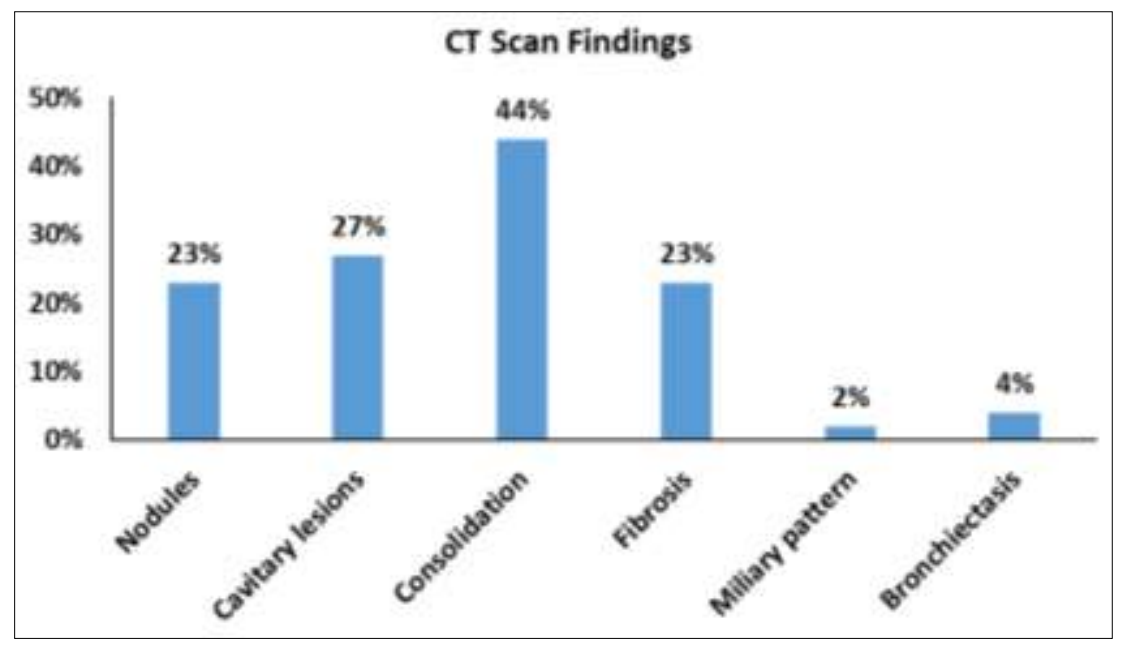

Fig 2: Type of TB patterns

\section{Discussion}

In this study out of 100 patients, most belonged to the age group 55-70 years $(46 \%)$ followed by $15-34$ years $(28 \%)$. The results of our study are similar to a study conducted in NRSMCH Kolkatta which revealed 47\%. Of the 100 cases included in this study for diagnosis of PTB predominantly males $(57 \%)$ compared to females $(43 \%)$ also there was predominance of males in study conducted by byArshad Altaf Bachh et al. $2010^{[1]} 66.7 \%$ were males and 33.3 were femalesand Sweta Madas et al. $2015^{[2]}$ reported $74 \%$ males and $26 \%$ females and Surendra k Sharma et al. $2015^{[3]} 65$ $\%$ males and $35 \%$ females. The most common symptom observed in this study was cough with or without expectoration $(74 \%)$ followed by fever $(52 \%)$. Which was noticed in a similar study conducted by Sweta Madas et al. $2015^{[4]}$ which showed $74 \%$ cough followed by fever (52\%) also in line with a study by Sanjay Avashia et al. $2016^{[5]}$ reported cough in $72.2 \%$ and fever in $69.7 \%$ of patients. Other symptoms observed was hemoptysis $(28 \%)$ in contrast study conducted by SwetaMadas et al. $2015^{\text {[6] }} 16 \%$ and

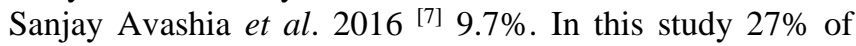
patients had cavitary lesions on the contrary in a study conducted by Sanjay Avashia et al. $2016^{[8]}$ reported cavity in $14 \%$ of cases.

$33 \%$ of cases had consolidation in a study conducted by

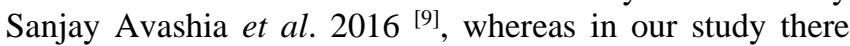
were $44 \%$ patients with consolidation.

Out of total 100 clinico-radiologcally suspected patients of PTB 51 patients were diagnosed as other diseases such as atypical pneumonia, aspergillus, Bronchogenic CA which are confirmed by culture \& HP study.

\section{Conclusion}

Our study mainly potrayed efficacy of BAL fluid CBNAAT in diagnosing TB and starting quick treatment in smear negative cases who are highly suspicious clinically and radiologically $\mathrm{TB}$ patients for microbiological evidence in era of drug resistant tuberculosis, as BAL fluid culture 
which is considered to be Gold standard but takes a long time for results. The results of our study also indicate how to minimize risk of starting of antitubercular treatment in patient in turn decresing the financial burden and the pill load who present similar to PTB clinically and radiologically.

Although at times there is a strong suspicion of PTB clinically or radiologically or both, physician should be cautious and rule out other possibilities i.e any other infective lesion or certain malignancies as ATT should be avoided in such cases. Also in cases of Old PTB patients both treated which appear with new onset of symptoms and radiololgically has certain lesions persisting or DRTB suspects BAL fluid CBNAAT not only helps in diagnosing but also shows wheather a previously treated TB patient is sensitive or resistant to Rifampicin. Our study focuses on clinicians dilemma in initiating Anti tubercular treatment in smear negative cases who are clinic radiologically suspected, BAL fluid CBNAAT proves to be a very useful tool in this case by readily diagnosing a patient and determining whether the patient is rifampicin sensitive or resistant and thus miminizing the number of patients in whom ATT is started only on clinic radiological basis and keep a follow up in relation to response to ATT both on clinical grounds and radiological grounds.

\section{References}

1. Arshad AltafBachh, Rahul Gupta, Inaamul Haq, Hanumant Ganapati Varudkar, Diagnosing sputum/ smear-negative pulmonary tuberculosis: Does fibreoptic bronchoscopy play a significant role?, lung India 2010;27(2):58-62.

2. Swetamadas, Mallikarjunnareddy. Role of induced sputum, bronchial aspirate and post FOB sputum in the diagnosis of sputum smear negative pulmonary tuberculosis. International Medical and Dental Research 2015, 1.

3. Evaluating the Diagnostic Accuracy of Xpert MTB/RIF Assay in Pulmonary Tuberculosis Surendra K Sharma, Mikashmi Kohli, Raj Narayan Yadav, Jigyasa Chaubey, Dinkar Bhasin, Vishnubhatla Sreenivas, Rohini Sharma, Binit K Singh, Plos 2015.

4. Swetamadas, Mallikarjunnareddy. Role of induced sputum, bronchial aspirate and post FOB sputum in the diagnosis of sputum smear negative pulmonary tuberculosis. International medical and dental research 2015, 1.

5. Sanjay Avashia, Satyadeo Choubey, Satyendra Mishra, Atul Kharate. To study the usefulness of cbnaat (cartridge based nuclear acid amplification test) in bal (bronchoalveolar lavage) samples in the diagnosis of smear-negative/non sputum producing patients with suspected tuberculosis. J Evolution Med Dent Sci 2016;5(1):55-59. doi: 10.14260/jemds/2016/13

6. Swetamadas, Mallikarjunnareddy. Role of induced sputum, bronchial aspirate and post FOB sputum in the diagnosis of sputum smear negative pulmonary tuberculosis. International medical and dental research 2015, 1

7. Sanjay Avashia, Satyadeo Choubey, Satyendra Mishra, Atul Kharate. To study the usefulness of cbnaat (cartridge based nuclear acid amplification test) in bal (bronchoalveolar lavage) samples in the diagnosis of smear-negative/non sputum producing patients with suspected tuberculosis. J Evolution Med Dent Sci 2016;5(1):55-59, doi: 10.14260/jemds/2016/13

8. Sanjay Avashia, SatyadeoChoubey, Satyendra Mishra, Atul Kharate. To study the usefulness of cbnaat (cartridge based nuclear acid amplification test) in bal (bronchoalveolar lavage) samples in the diagnosis of smear-negative/non sputum producing patients with suspected tuberculosis. J Evolution Med Dent Sci 2016;5(1):55-59. doi: 10.14260/jemds/2016/13

9. Sanjay Avashia, Satyadeo Choubey, Satyendra Mishra, Atul Kharate. To study the usefulness of cbnaat (cartridge based nuclear acid amplification test) in bal (bronchoalveolar lavage) samples in the diagnosis of smear-negative/non sputum producing patients with suspected tuberculosis. J Evolution Med Dent Sci 2016;5(1):55-59. doi: 10.14260/jemds/2016/13 